\title{
NON-SILICIOUS, DOUBLY REFRACTILE CRYSTALS IN THE GIANT CELLS OF TUBERCULOSIS \\ BY
}

\author{
CHARLES P. FARTHING AND BASIL G. PICKLES \\ From the Departments of Pathology and Gynaecology, King's College Hospital, London
}

(RECEIVED FOR PUBLICATION JANUARY 26, 1957)

The diagnosis of silicious or talc granuloma has often been made upon histological evidence unsupported by any chemical tests. Such a diagnosis would appear to be confirmed by finding non-caseating "tubercle" follicles and doubly refractile crystalline particles in or near multinucleate giant cells, with a negative staining reaction for acid-fast bacilli.

Five cases of silicious granuloma in the Fallopian tube were described by Roberts (1947), appendicectomy having been performed previously in each of these cases, but in only one was the diagnosis proved by chemical tests.

Smith (1945) recorded an unusual case of carcinoma of the body of the uterus together with tuberculosis of the endometrium and Fallopian tubes. After the publication of the article by Roberts (1947), Smith (1948) re-examined his sections and found doubly refractile bodies within giant cells, which convinced him that the lesions were silicious and not tuberculous. No chemical tests were performed to confirm or refute this amended diagnosis.

Potts (1951) reported two cases of tuberculous salpingitis which showed typical caseating tubercle follicles and multinucleate giant cells. Sections from both the cases contained strongly doubly refractile crystalline particles within giant cells, which morphologically resembled talc. When subjected to an acid-solubility test and microincineration, the crystals were shown not to be silicious. Although no acid-fast bacilli were discovered when similar sections were specially stained, the lesions were considered to be tuberculous and not talc granulomata.

Burne (1953) attempted to verify the association of laminated (Schaumann) bodies and doubly refractile bodies with tuberculous salpingitis. Eleven of the $\mathbf{4 2}$ cases reviewed showed acidsoluble, i.e., non-silicious, birefringent crystals, mostly in multinucleate giant cells. The absence of silica was confirmed in two cases by micro- incineration of sections. In addition, one case of talc granuloma was discovered, silica being identified by chemical tests.

A tubal pregnancy occurring in a tuberculous uterine tube was reported by Berenbaum and Korn (1954). The tuberculous lesion contained many doubly refractile crystals within giant cells, which were shown by histochemical analysis to consist of calcium carbonate.

Pearse (1953) shows illustrations of birefringent crystalline inclusions within multinucleate giant cells in sections from a human lymph node.

This article briefly describes a case in which the histological appearance of the lesion closely simulated that of a talc granuloma, but was later proved to be tuberculous. Findings from a series of histochemical investigations carried out on Fallopian tubes and other tuberculous organs are recorded and commented upon.

\section{Case Report}

Mrs. R. was first seen in 1956 complaining of four years' infertility. A year previously infertility investigations had been carried out; at that time a tubal insufflation and curettage had, apparently, revealed no abnormality. A salpingogram was performed which showed dilated uterine tubes, both blocked at the fimbrial ends.

At operation the naked-eye appearance of the Fallopian tubes was at first sight completely normal. The fimbriae and serous coat showed no abnormality even on close inspection. However, on attempting to pass a probe through the abdominal ostia it became apparent that there were very tight stricture; at the bases of the fimbriae. The width of the strictures was no wider than a thin piece of string. On making incisions through the narrow strictures it was seen that the lumen of each tube was somewhat dilated and the wall of the tube somewhat oedematous and thickened. There were no other strictures visible or palpable along the course of the tubes. A piece of tissue was removed from the site of one stricture and bilateral salpingostomy was performed. 


\section{Pathology}

Portions of Fallopian tube were used for histological examination, guinea-pig inoculation, and culture for tubercle bacilli on egg media. A section of the tubal tissue stained with haematoxylin and eosin showed non-caseating tubercle follicles with multinucleate giant cells, many of the latter containing crystalline particles. These crystals were strongly doubly refractile when examined in polarized light. No tubercle bacilli were found, using both the ZiehlNeelsen and auramine-rhodamine-phenol techniques.

From these findings a diagnosis of talc granuloma was made. Histochemical tests were then performed in an attempt to confirm the silicious nature of the anisotropic crystals. The results proved that the doubly refractile crystalline particles were nonsilicious, and therefore not talc. The tubal lesions were shown to be tuberculous in nature by the growth of $M$. tuberculosis both on the egg media and in the inoculated guinea-pig.

Having found a large number of intracellular. doubly refractile crystals in a proved case of tuberculosis, it was decided that sections from other cases of tuberculous salpingitis should be examined to ascertain the incidence of these inclusions.

\section{Material and Methods}

Sections from the cases of tuberculous salpingitis seen in the hospital during the past 10 years were examined. Thirty such cases were reviewed, and, in addition, a case reported as "pseudo-tuberculous foreign-body reaction in the Fallopian tube due to talc" was examined, which, with the one already described, made a total of thirty-two. Besides the original slides, one or more blocks of wax-embedded tubal tissue were available from every case. The slides were cleaned with xylol to remove any wax which is itself brilliantly doubly refractile. The sections were "screened" under low power in polarized light, using a Swift polarizing microscope. It was found easiest to perform this manœuvre with the planes of the polarizer and analyser not quite fully crossed. This enabled any birefringent particles to be picked out easily, and yet gave sufficient general illumination to make it possible for the relationship of the particles to the surrounding tissues to be seen. Only those doubly refractile particles seen to lie within multinucleate giant cells were recorded as being significant for this investigation. Only by using this strict criterion was it possible to exclude material not derived from the specimen, for many doubly refractile particles were seen other than those in giant cells, possibly introduced during the processing of the seztions.

Six new sections were cut from each block corresponding to the sections found to contain doubly refractile inclusions. Each set of six sections was used as follows :

(1) One section was stained with haematoxylin and eosin and mounted under a coverslip in the usual way.
(2) Two sections had the wax dissolved away, were lightly stained with aqueous methylene blue, and left uncovered.

(3) The remaining three sections were kept as "spares."

The two lightly stained sections were subjected to screening in polarized light after the application of a drop of xylol and a thin coverslip. The position of any birefringent particles in multinucleate giant cells was noted on the vernier scales of the microscope. If no inclusions were found, the three spare sections were similarly stained and examined.

When birefringent particles were discovered in a section the coverslip was removed, and, after evaporation of the xylol, the section itself covered with dilute hydrochloric acid $(\mathrm{N} / 1)$. After 30 minutes the acid was gently rinsed off with distilled water and the section allowed to dry. The areas which had contained birefringent particles were then re-examined in polarized light to ascertain whether the inclusions had been dissolved.

The second slide containing intracellular crystals was subjected to micro-incineration, using the technique described by Irwin (1934). A small electrically heated muffle-furnace was utilized, the slides being placed on a stainless steel tray and gradually heated to $600^{\circ}$ C. (dull red heat), this temperature being maintained for one hour. After cooling, the areas containing birefringent particles were re-examined, first in polarized light and then by reflected light. The appearance of the incinerated inclusions was noted and the ash then treated with dilute hydrochloric acid $(\mathrm{N} / 1)$ for 30 minutes. If the inclusion ash had not been dissolved by the dilute acid, the effect of concentrated hydrochloric acid was also investigated.

To act as a known positive control, a sample of surgical talc was fixed in formol saline. and embedded in wax in exactly the manner used for tissues. Six sections were prepared, two of which were subjected to the tests described. Material containing silica (talc in this instance) was found to be insoluble in dilute and concentrated acid, both before and after microincineration, the latter process altering neither the appearance of the crystals nor their birefringence.

\section{Results}

Seventeen of the cases investigated revealed multinucleate giant cells containing birefringent particles, the two "talc granuloma" cases being included among these. The number of separate inclusions encountered in the "positive" slides ranged from one to more than 100 . In every case the birefringent particles in the giant cells presented either as single, irregularly shaped, but rather rounded crystals, or as round or oval aggregates of similar material. The size of the individual particles was variable, reaching a maxi- 
mum of about $40 \mu$. By transmitted light the crystals appeared colourless or a very pale yellow. Viewed in polarized light, with the planes crossed, they became brilliantly doubly refractile, many of the larger crystals exhibiting a play of colours (Figs. 1 and 2).

On comparison, the crystals of the talc control were as variable in size, but morphologically were much less rounded. Talc was found also to be much less birefringent, many of the larger crystals being doubly refractile only at the edges.

All the birefringent inclusions were found to be soluble in dilute hydrochloric acid, leaving round or oval deficiencies in the giant cells corresponding to the size and shape of the crystals dissolved away.

Micro-incineration converted the birefringent crystals into white amorphous material, the particles of which were approximately the size of the original crystals, showing much reduced or absent birefringency. Most of these altered particles dissolved rapidly in dilute hydrochloric acid, but a few required concentrated acid.

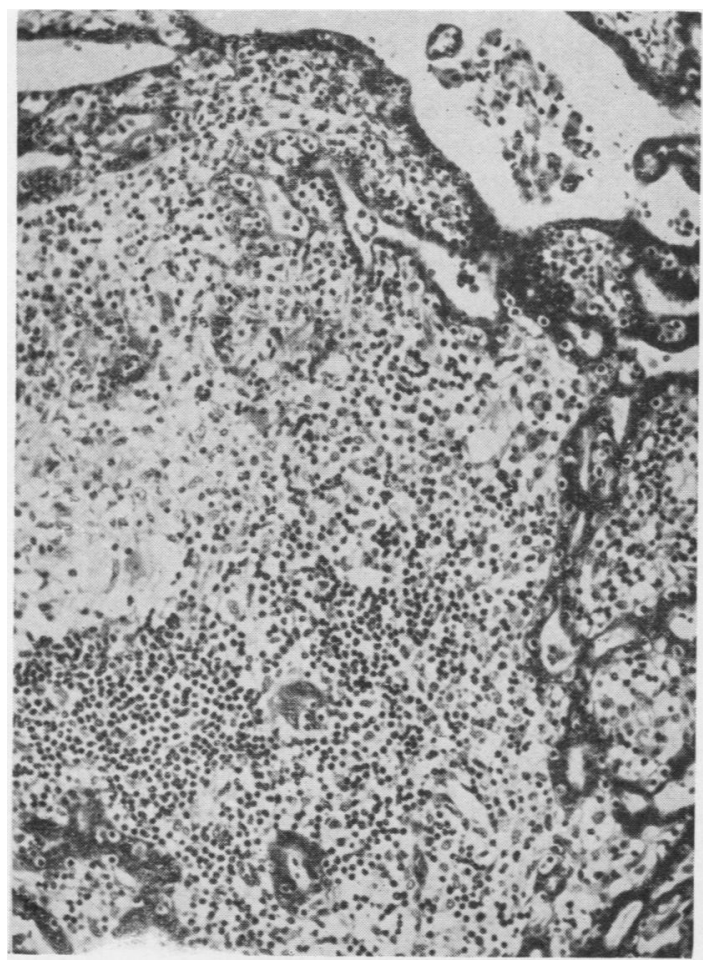

Fig. 1.-Section of Fallopian tube photographed in ordinary light showing a non-case at ing tubercle follicle with multinucleate giant cells. (Haematoxylin and eosin, $\times 130$.)
From these investigations it was concluded that the material in the multinucleate giant cells was not silicious. This included the material in both the cases previously described as being talc granulomata.

Relationship of Inclusions to Caseation.-The 32 cases were divided into those showing and those not showing caseation. Doubly refractile inclusions were found in 8 of the 11 with no caseation and in only 9 of the 21 with caseation.

Similarly, when the frequency of the inclusions was investigated in the 17 positive cases, it was usually the non-caseating lesion which contained the large number of birefringent inclusions.

\section{Intracellular Birefringent Crystals in Other Organs}

As over half of the tuberculous Fallopian tubes were found to contain doubly refractile crystals, an attempt was made to discover whether this finding was common to tuberculous lesions in other tissues. Sections from 10 cases

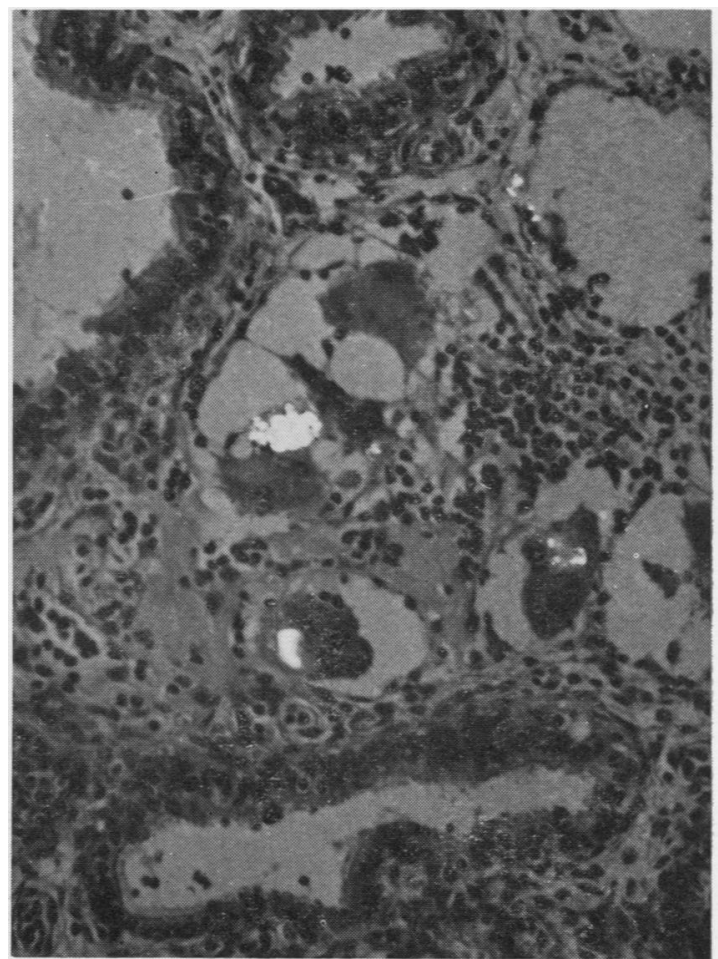

FIG. 2.-Part of same section as Fig. 1 photographed in polarized light with planes not quite fully crossed. Four giant cells contain doubly refractile inclusions. (Haematoxylin and eosin, $\times 200$.) 
each of tuberculous lymph nodes, kidney, and epididymis were studied. Lung tissue was excluded because of the possibility of exogenous silica being present. The methods used for screening, followed by chemical tests, were similar to those used for the Fallopian tubes, with the following results:

\begin{tabular}{|c|c|c|c|c|c|c|}
\hline Lymph nodes & 10 & \multicolumn{5}{|c|}{10 cases studied, 3 contained crystals } \\
\hline Kidney & 10 &.. & , , & 8 & , & , \\
\hline Epididymis & 10 & .. & ,. & 6 & , &. \\
\hline
\end{tabular}

This means that $17(57 \%)$ of the 30 cases examined showed birefringent crystalline inclusions in giant cells almost identical with those found in the Fallopian tubes. They showed the same variation in number, but were usually smaller and more widely scattered through the sections (Fig. 3).

Acid-solubility tests and micro-incineration showed that the crystals behaved exactly as did those from the tubes, and therefore were also non-silicious.

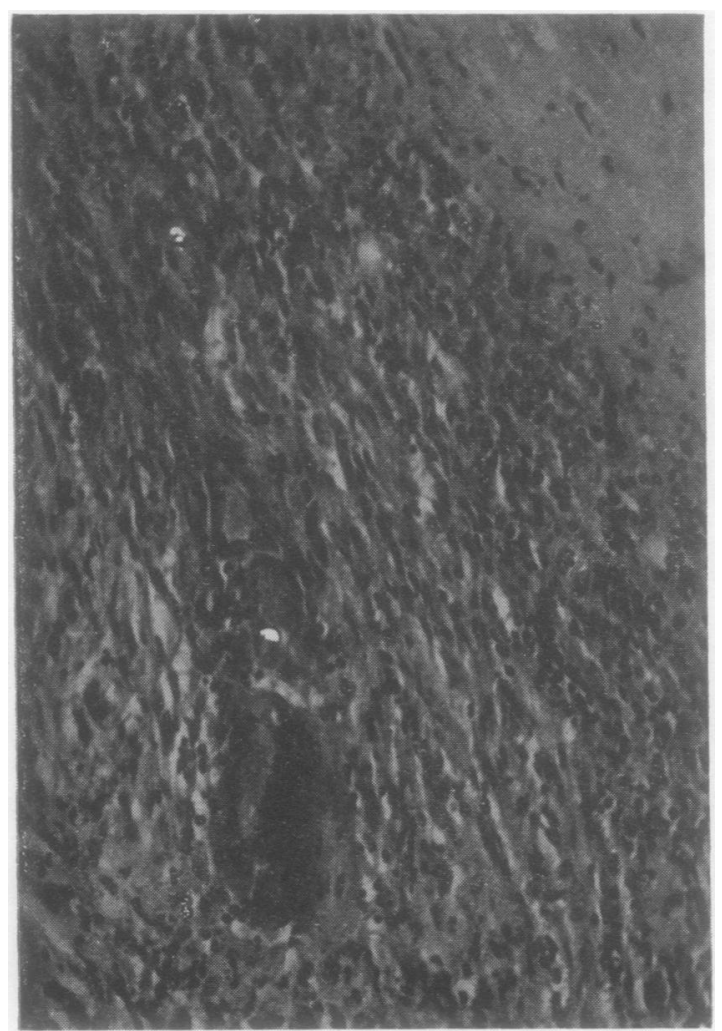

FIG. 3.-Section of tuberculous lymph node, photographed in polarized light. Two giant cells contain small birefringen inclusions. (Haematoxylin and eosin, . 200.)

\section{Chemical Composition of the Crystals}

A limited histochemical analysis was attempted using only sections from a case of tuberculous salpingitis showing numerous inclusions.

The crystals gave a strongly positive reaction to von Kossa's test, thus demonstrating them to be either phosphate or carbonate. When glacial acetic acid was applied to the inclusions by running it under a coverslip, the crystals were seen to dissolve without any bubble $\left(\mathrm{CO}_{2}\right)$ formation. The crystalline material was therefore, in all probability, a phosphate.

Pearse (1953) states that nearly all deposits of insoluble carbonate or phosphate in animal tissue are calcium salts. It can be presumed therefore that the material investigated was a calcium phosphate. Some confirmation of this was obtained by subjecting a preparation of known calcium phosphate crystals to all the tests described here. with results identical with those obtained with the inclusions.

\section{Comment}

Burne (1953) investigated 41 cases of tuberculous salpingitis and found Schaumann bodies in $29 \%$ and doubly refractile crystalline inclusions in $27 \%$ of them. In the present series similar crystals were found within multinucleate giant cells in $55 \%$ of all the cases studied (a total of 62). This result surely demonstrates that doubly refractile crystals within multinucleate giant cells occur very frequently, not only in tuberculous salpingitis but also in tuberculous lesions of other organs.

Potts (1951) found that birefringent crystalline inclusions were scanty or absent when caseation was present. The present findings seem to confirm this. This increased frequency in noncaseating tuberculous lesions has, on occasions. possibly led to a mistaken diagnosis of talc granuloma being made. If, therefore, the diag nosis of silicious granuloma is to be made with certainty, the quick and easily performed acidsolubility test should be carried out on the crystals. This is not put forward as an original suggestion, for Potts made the observation in his paper, but it is reiterated and emphasized here because it seems to be of such importance.

\section{Summary}

A case of tuberculous salpingitis is described on which a diagnosis of talc granuloma had been made originally.

Investigation of 32 cases of tuberculous salpingitis showed sections of the lesions from 17 of them 
to contain birefringent, non-silicious crystalline inclusions within multinucleate giant cells.

A further 30 cases with tuberculosis of other organs (excluding lung) similarly investigated showed sections from 17 of them to contain similar crystalline inclusions.

The birefringent crystals in giant cells were found to be more common when caseation was absent, confirming the findings of other workers, and in one case were shown to consist probably of calcium phosphate.

The necessity of performing a simple acidsolubility test on the birefringent inclusions before making a diagnosis of silicious granuloma is emphasized.
We are indebted to Mr. J. H. Peel for permission to publish the case; to Professor H. A. Magnus for his advice and encouragement ; and to Mr. G. Harwood for the photomicrographs and technical assistance.

\section{REFERENCES}

Berenbaum, M. C., and Korn, G. W. (1954). J. Obstet. Gynaec. Brit. Emp., 61, 351.

Burne, J. C. (1953). J. Path. Bact., 65, 101.

Irwin, D. A. (1934). Canad. med. Ass. J., 31, 135.

Pearse, A. G. E. (1953). Histochemistry, pp. 378-383. Churchill, London.

Potts, R. E. (1951). J. clin. Path., 4, 333.

Roberts, G. B. S. (1947). Brit. J. Surg., 34, 417.

Smith, G. H. (1945). J. Obstet. Gynaec. Brit. Emp., 52, 189.

- (1948). Brit. med. J., 1, 1078. 